\title{
3 Research Suare \\ Feasibility and Efficacy of Prehospital Esmolol for Refractory Ventricular Fibrillation
}

Casey Patrick ( $\nabla$ cpatrick@mchd-tx.org )

Montgomery County Hospital District EMS

\section{Remle Crowe}

HCA Houston Healthcare Kingwood

\section{Brad Ward}

Montgomery County Hospital District EMS

Ali Mohammed

HCA Houston Healthcare Kingwood

Kelly Rogers Keene

Baylor College of Medicine

Robert Dickson

Montgomery County Hospital District EMS

\section{Research Article}

Keywords: Out of Hospital Cardiac Arrest, Esmolol, Refractory Ventricular Fibrillation, Emergency Medical Services, Prehospital, Cardiac Arrest

Posted Date: September 1st, 2021

DOI: https://doi.org/10.21203/rs.3.rs-752531/v1

License: (c) (1) This work is licensed under a Creative Commons Attribution 4.0 International License.

Read Full License 


\section{Abstract}

Background: We aimed to assess the feasibility of prehospital bolus dose esmolol for patients with outof-hospital cardiac arrest (OHCA) and refractory ventricular fibrillation (RVF) treated by a single, highvolume, ground-based emergency medical services (EMS) agency.

Methods: This retrospective observational study evaluated EMS patients with RVF treated from June 10, 2017 to June 10, 2020. Esmolol (0.5mg/kg bolus) was added to the RVF protocol at the mid-point of the study period on December 10, 2018.

Results: We analyzed 61 patients treated with prehospital esmolol and 63 patients treated without esmolol. Median time from EMS arrival to esmolol administration was 17 minutes (interquartile range: 13-22 minutes). Prehospital return of spontaneous circulation (ROSC) was higher in the esmolol group compared to the control group, though statistical significance was not reached ( $38 \%$ versus $24 \%, p=0.09$ ). Overall, few patients survived to 24 hours (esmolol $n=15$, pre-esmolol $n=16$ ) and fewer survived to hospital discharge (esmolol $n=5$, pre-esmolol $n=5$ ), precluding stable statistical comparisons.

Conclusion: Our findings suggest that esmolol is feasible in the prehospital setting and may lead to increased ROSC. Further large-scale studies are needed to determine the effect of prehospital esmolol for RVF as it relates to survival to hospital discharge.

Trial Registration: This study was approved by the institutional review board at The Baylor College of Medicine and a waiver of informed consent was granted - protocol number $\mathrm{H}-48383$.

\section{Background}

Refractory ventricular fibrillation (RVF) is often defined as cardiac arrest with persistent ventricular fibrillation despite initiation of American Heart Association Advanced Cardiac Life Support (ACLS), three defibrillations, three doses of $1 \mathrm{mg}$ epinephrine and amiodarone administration (1-2). From a prehospital perspective, this is a specific subset of out-of-hospital cardiac arrest (OHCA), unlike classic electrical storm described in the cardiology literature (3-4), with dismal prognoses and outcomes despite current standard care (5-6). Early defibrillation is known to increase survival in patients with OHCA who present with initial shockable rhythms (7-8), amiodarone potentially increases survival to hospital admission (9), but no pharmacologic treatments have prospectively demonstrated increases in survival with neurologically intact outcomes (10). However, two small hospital-based observational studies (11-12) and earlier animal studies (13-15) have suggested increased survival in RVF with the addition of the ultrashort acting beta-blocker, esmolol, to standard ACLS care. RVF is thought to result from adrenergic surge, often secondary to acute coronary occlusion and myocardial ischemia (16), with esmolol providing benefit by selectively blocking beta- 1 adrenergic receptors and decreasing myocardial oxygen demands. 
RVF is often first encountered outside the emergency department or hospital environment, making esmolol an attractive potential treatment option for paramedic providers. Within the prehospital literature, however, the feasibility of esmolol use in RVF patients has not been evaluated in the EMS environment. In this study, the feasibility of prehospital bolus dose esmolol in OHCA patients progressing to RVF was assessed by paramedics within a single, high-volume, ground-based EMS agency.

\section{Methods}

\section{Study Design and Setting}

We conducted a retrospective observational analysis of all OHCA patients with refractory ventricular fibrillation/ encountered by a large, suburban, county-based EMS service in Texas between June 10, 2017 and June 10, 2020. This study was approved by the institutional review board at The Baylor College of Medicine and a waiver of informed consent was granted.

This ground-based EMS agency employs approximately 250 advanced life support providers and more than 1,000 emergency medical technicians (EMTs). In a service area encompassing 1,100 square miles, the agency responds to more than 70,000 annual calls for service.

Prior to December 10, 2018, the treatment protocol for RVF followed standard advanced cardiac life support recommendations including high-quality CPR, epinephrine administration ( $1 \mathrm{mg} \times 3$ doses, Q5min), three defibrillations (200J), anti-arrhythmic administration and advanced airway management with either an endotracheal tube or supraglottic device. The addition of esmolol $(0.5 \mathrm{mg} / \mathrm{kg}$ bolus) to the cardiac arrest treatment protocol for RVF occurred at the midpoint of our data collection period on December 10, 2018. Dual sequential defibrillation (DSD) was available and encouraged, but not required, throughout the entire study period.

The EMS protocol developed for bolus dose esmolol used in this study is noted in Figure 1. Paramedics initially identified RVF and following the third defibrillation, esmolol was available for use after the study midpoint at a dose of $0.5 \mathrm{mg} / \mathrm{kg}$. Due to relatively short transport times, we elected to administer only an esmolol bolus without the initiation of a continuous drip.

As part of the update of this protocol at the midpoint of the study, 250 paramedics underwent a mandatory 2-hour training session that included a review of cardiac arrest physiology, focused didactic instruction on the pathophysiology and clinical findings in RVF along with an introduction to specifics of esmolol pharmacology and an introduction to the esmolol treatment protocol (Figure 1). The training sessions occurred approximately one month prior to protocol deployment. This knowledge was then reinforced by a dedicated RVF podcast, produced in-house, which was available and promoted for the duration of the study period. Providers demonstrated an understanding of RVF and the esmolol treatment protocol through both written and psychomotor examinations at the conclusion of the mandatory training session. 
Patients or the public were not involved in the design, or conduct, or reporting, or dissemination plans of our research.

The prehospital electronic patient care record (ePCR) system was queried for all OHCA patients with three or more defibrillations. EMS patient care records were then individually and independently reviewed by two physician authors (CP and RD) to determine presence of true RVF as defined by shock resistance with persistent ventricular fibrillation, and no degeneration into pulseless electrical activity (PEA) or asystole, during the initial ACLS treatment phase. Cardiac arrests with traumatic etiologies were excluded from the analysis.

\section{Measures}

Data elements were abstracted directly from the prehospital ePCR and hospital electronic medical records by a two-person expert review panel consisting of one physician (CP) and one paramedic (BW) using a standardized data collection form. The study variables included patient demographic information, medications, interventions, and cardiac rhythm present throughout the prehospital encounter.

Outcome measures included presence or absence of ROSC during the EMS encounter, 24-hour hospital survival, and survival to hospital discharge. ROSC was ascertained using the prehospital electronic patient care record while survival measures were obtained from the hospital electronic health record.

\section{Statistical Analysis}

Descriptive statistics were calculated with frequencies and percentages for categorical variables and continuous variables summarized using medians and interquartile ranges (IQR). Rates of ROSC were compared using Chi-square tests. Multivariable logistic regression modelling was used to assess the association between esmolol and outcome variables while controlling for patient age, sex, and initial rhythm. All analyses were completed using Stata IC Version 15.1 (StataCorp LLC, College Station, TX).

\section{Results}

Prior to the addition of esmolol to the EMS protocol, we identified 87 patients with 3 or more defibrillations in the out-of-hospital setting, of which 24 were excluded after full review as RVF was not present. After the addition of esmolol, there were 105 patients with at least 3 defibrillations of which 33 were deemed non-RVF and excluded after review. Two patients with traumatic arrest etiologies were excluded and 9 eligible patients that did not receive esmolol were excluded. The analysis sample consisted of 61 patients treated with esmolol and 63 patients encountered before esmolol was introduced into the EMS protocol (Fig. 2).

Patient and encounter characteristics were similar for patients who did and did not receive esmolol (Table 1). The median age was 67 years in both groups and approximately $28 \%$ were female. Bystander CPR was noted in more than $75 \%$ of cases. Approximately $87 \%$ of patients in both groups presented with an initial rhythm of either VF or VT. Median time to the first dose of epinephrine was 4 minutes in both 
groups. The median number of epinephrine doses was slightly higher in the group that did not receive esmolol ( 5 doses) compared to the group that received esmolol (3 doses). The median EMS scene time was 35 minutes (IQR: 30-41 minutes) in the esmolol group compared to 32 minutes (IQR: 26-41 minutes) in the group that did not receive esmolol. 
Table 1

Patient and EMS encounter characteristics.

\begin{tabular}{|c|c|c|c|}
\hline & $\begin{array}{l}\text { Esmolol } \\
\mathrm{N}=61\end{array}$ & $\begin{array}{l}\text { No Esmolol } \\
\mathrm{N}=63\end{array}$ & p-value \\
\hline \multicolumn{4}{|l|}{ Age, years } \\
\hline Median (IQR) & $67(57-76)$ & $67(57-77)$ & 0.60 \\
\hline Sex & & & 0.93 \\
\hline Female & $27.9 \%(17)$ & $28.6 \%(18)$ & \\
\hline Male & $72.1 \%(44)$ & $71.4 \%(45)$ & \\
\hline Race & & & 0.56 \\
\hline White & $78.7 \%(48)$ & $85.7 \%(54)$ & \\
\hline Black & $4.9 \%(3)$ & $3.2 \%(2)$ & \\
\hline Hispanic & $13.1 \%(8)$ & $6.4 \%(4)$ & \\
\hline Other/Unknown & $3.3 \%(2)$ & $4.8 \%(3)$ & \\
\hline Bystander CPR & & & 0.91 \\
\hline Yes & $77.1 \%(47)$ & $76.2 \%(48)$ & \\
\hline No & $22.9 \%(14)$ & $23.8 \%(15)$ & \\
\hline Initial rhythm & & & 0.24 \\
\hline VF & $83.6 \%(51)$ & $82.5 \%(52)$ & \\
\hline VT & $3.3 \%(2)$ & $4.8 \%(3)$ & \\
\hline PEA & $3.3 \%(2)$ & $9.5 \%(6)$ & \\
\hline Asystole & $9.8 \%(6)$ & $3.2 \%(2)$ & \\
\hline Dual sequential defibrillation & & & 0.46 \\
\hline Yes & $24.6 \%(15)$ & $19.0 \%(12)$ & \\
\hline No & $75.4 \%(46)$ & $81.0 \%(51)$ & \\
\hline \multicolumn{4}{|l|}{ Number of defibrillations } \\
\hline Median (IQR) & $5(4-7)$ & $6(5-9)$ & 0.12 \\
\hline Total doses of epinephrine & & & $<0.01$ \\
\hline Median (IQR) & $3(2-4)$ & $5(4-6)$ & \\
\hline
\end{tabular}




\begin{tabular}{|llll|}
\hline & $\begin{array}{l}\text { Esmolol } \\
\mathbf{N}=61\end{array}$ & $\begin{array}{l}\text { No Esmolol } \\
\mathbf{N}=63\end{array}$ & p-value \\
\hline Median (IQR) & $4(3-5)$ & $4(2-6)$ & 0.79 \\
\hline EMS scene time, minutes & & & \\
\hline Median (IQR) & $35(30-41)$ & $32(26-41)$ & 0.21 \\
\hline ED transport & & & 0.01 \\
\hline Yes & $62.3 \%(38)$ & $82.5 \%(52)$ & \\
\hline No & $37.7 \%(23)$ & $17.5 \%(11)$ & \\
\hline EMS transport time, minutes & & & 0.51 \\
\hline Median (IQR) & $10.5(8-15)$ & $12(8.5-17.5)$ & \\
\hline
\end{tabular}

Table 2

Prehospital ROSC, 24-hour survival and survival to hospital discharge.

\begin{tabular}{|llll|}
\hline & $\begin{array}{l}\text { Esmolol } \\
\text { N=61 }\end{array}$ & $\begin{array}{l}\text { No Esmolol } \\
\mathbf{N}=63\end{array}$ & p-value \\
\hline Prehospital ROSC & & & 0.09 \\
\hline Yes & $37.7 \%(23)$ & $23.8 \%(15)$ & \\
No & $62.3 \%(38)$ & $76.2 \%(48)$ & \\
\hline $24-$ hour survival & & & 0.92 \\
\hline Yes & $24.6 \%(15)$ & $25.4 \%(16)$ & \\
No & $75.4 \%(46)$ & $74.6 \%(47)$ & \\
\hline Survival to hospital discharge & & & 0.96 \\
Yes & $8.2 \%(5)$ & $7.9 \%(5)$ & \\
No & $91.8 \%(56)$ & $92.1 \%(58)$ & \\
\hline
\end{tabular}

The median on scene arrival time to esmolol administration was 17 minutes (IQR: 13-22 minutes). After esmolol administration $38 \%(n=23)$ of patients achieved prehospital ROSC compared to $24 \%(n=15)$ of patients that did not receive esmolol, though this difference did not reach statistical significance. Survival at 24 hours was similar for those who received esmolol $(25 \%, n=15)$ and those who did not receive esmolol $(25 \%, n=16)$. In total, 5 patients ( $8 \%)$ from the esmolol group and 5 patients ( $8 \%)$ from the group that did not receive esmolol survived to hospital discharge. 
After adjusting for patient age, sex, and initial rhythm, there was a two-fold increase in odds of ROSC during the EMS encounter (OR: 1.99, 95\% Cl: 0.89-4.47) for patients treated with esmolol compared to patients who were not administered esmolol, though statistical significance was not reached (Fig. 3).

\section{Discussion}

In this pragmatic study of patients with RVF encountered by EMS, administering esmolol in the out-ofhospital setting was feasible and non-inferior to standard ACLS treatment. Though no statistical differences were noted, there appeared to be a trend towards higher ROSC for RVF patients administered esmolol. Overall, few patients survived to 24 hours and fewer survived to hospital discharge, making comparisons between groups challenging.

More patients who were administered prehospital esmolol experienced ROSC compared to those treated before the introduction of esmolol into the EMS protocol for RVF. Two in-hospital studies evaluating ED administration of esmolol for patients with OHCA and RVF also identified higher rates of ROSC in the esmolol group $(11,12)$. While ROSC does not directly translate to survival, re-establishing circulation and perfusion in the prehospital setting prior to patient transport may help preserve neurologic function while allowing EMS to safely route the patient to appropriate continued care and advanced interventions. Recently, extracorporeal membrane oxygenation assisted CPR programs have shown benefit for RVF (19), however these programs largely take place in specific, specialized hospital settings. Early ROSC and immediate EMS transport of RVF patients to extracorporeal membrane oxygenation assisted CPR capable facilities may be beneficial within some systems of care where these resources exist.

Overall survival of patients with RVF was low throughout the study period. These findings are consistent with overall poor outcomes observed for patients presenting with RVF (4-6). Prehospital OHCA patients presenting with RVF are a complex, heterogenous patient population with a multi-faceted clinical management pathway. This complexity makes true standardization between patients exceedingly difficult in the EMS setting. For example, while available and encouraged throughout the study period, only $20 \%$ of RVF patients in both the pre- and post-esmolol groups received DSD. While the current evidence for the efficacy of DSD in RVF is mixed $(17,18)$, it is possible that increased DSD use in conjunction with esmolol administration could potentially lead to improved outcomes. Also, due to having relatively short EMS transport times and to minimize logistical complexities, we elected to defer initiation of an esmolol drip within our protocol. Two hospital-based studies suggesting improved outcomes with esmolol in OHCA with RVF $(11,12)$ used a $0.5 \mathrm{mg} / \mathrm{kg}$ loading dose bolus followed by a 0 $100 \mathrm{mcg} / \mathrm{kg} / \mathrm{min}$ infusion. Future large-scale prospective EMS investigations of both the pairing of DSD with esmolol in addition to a protocolized initiation of an esmolol infusion following the initial bolus dose are warranted.

Finally, the role of prehospital administered epinephrine in OHCA for patients with RVF is unclear. Use of epinephrine for OHCA in general has been questioned with the results of the PARAMEDIC-2 trial (20) showing no increase in 30-day neurologically intact survival when using epinephrine in OHCA, as 
compared to placebo. Additionally, in consideration of patients with RVF, it is worth noting that fibrillated myocardium has significantly increased oxygen consumption relative to normal $(21,22)$, which exogenous epinephrine likely increases via beta adrenergic stimulation. With the underlying pathophysiology of RVF believed to be a result of endogenous catecholamine surge in response to active myocardial ischemia (16), it bears consideration that an early esmolol administration protocol, without exogenous epinephrine, may be beneficial in OHCA, specifically for patients presenting with shockable rhythms and those who progress to RVF. In our study, the time from EMS arrival to esmolol administration was nearly 20 minutes, further evaluation of earlier B-blockade warrants future investigation.

This study was limited by its retrospective nature, small sample size and due to the fact that patients were treated within a single ground-based EMS agency. Further, small sample size and lack of standardization of DSD limited the ability to study the effects of prehospital esmolol on overall RVF morbidity and mortality. Randomized studies with larger sample sizes are needed to further investigate the effects of prehospital esmolol on patient morbidity and mortality in patients with RVF.

\section{Conclusions}

This study is the first ground-based EMS prehospital evaluation of the use of esmolol in OHCA patient with RVF. While esmolol use was demonstrated to be feasible in the prehospital setting, under this protocol, there was no change in neurologically intact survival to hospital discharge in RVF patients treated with esmolol as compared to those treated with standard ACLS. Future work must be directed at further prospective, randomized evaluations of a potential role for esmolol in prehospital RVF treatment.

\section{Abbreviations}

OHCA - Out of Hospital Cardiac Arrest

RVF - Refractory Ventricular Fibrillation

EMS - Emergency Medical Services

ROSC - Return of Spontaneous Circulation

ACLS - Advanced Cardiac Life Support

EMT - Emergency Medical Technician

DSD - Dual Sequential Defibrillation

ePCR - Electronic Patient Care Record

PEA - Pulseless Electrical Activity

IQR - Interquartile Range 


\section{Declarations}

\section{Ethics Approval and Consent to Participate}

This study was approved by the institutional review board at The Baylor College of Medicine and a waiver of informed consent was granted - protocol number $\mathrm{H}-48383$.

\section{Consent for Publication}

Not applicable

\section{Availability of Data and Materials}

Deidentified participant data and applicable protocols are available from the corresponding author: cpatrick@mchd-tx.org

\section{Competing Interests}

The authors have no competing interests to disclose.

\section{Funding}

The authors have no funding sources to disclose.

\section{Author's Contributions}

All authors have made substantial contributions to the conception and design of the study (CP, $R C, B W, K K, R D)$, acquisition of data (CP,BW,AM), analysis and interpretation of data (CP, BW, RC), and drafting/revising it critically for important intellectual content (CP,RC).

\section{Acknowledgements}

Not applicable

\section{References}

1. Miraglia D, Miguel LA, Alonso W. Esmolol in the management of pre-hospital refractory ventricular fibrillation: A systematic review and meta-analysis. Am J Emerg Med. 2020;38:1921-34.

2. Koster RW, Walker RG, Chapman FW. Recurrent ventricular fibrillation during advanced life support care of patients with prehospital cardiac arrest. Resuscitation. 
3. Gao D, Sapp JL. Electrical storm: definitions, clinical importance, and treatment. Curr Opin Cardiol. 2013;28:72-9.

4. Eifling M, Razavi M, Massumi A. The evaluation and management of electrical storm. Tex Heart Inst J. 2011;38:111-21.

5. Benjamin EJ, Muntner P, Alonso A, et al. Heart Disease and Stroke Statistics-2019 Up- date: A Report From the American Heart Association. Circulation. 2019;139: e56-528.

6. Sakai T, Iwami T, Tasaki O, et al. Incidence and outcomes of out-of-hospital cardiac arrest with shockresistant ventricular fibrillation: data from a large population-based cohort. Resuscitation. 2010;81:95661.

7. Al-Khatib SM, Stevenson WG, Ackerman MJ, et al. 2017 AHA/ACC/HRS guideline for management of patients with ventricular arrhythmias and the prevention of sudden cardiac death: a report of the American College of Cardiology Foundation/American Heart Association Task Force on Clinical Practice Guidelines and the Heart Rhythm Society. Circulation. 2018;138:e272-391.

8. Wang $\mathrm{CH}$, Huang $\mathrm{CH}$, Chang WT, et al. Biphasic versus monophasic defibrillation in out-of-hospital cardiac arrest: a systematic review and meta-analysis. Am J Emerg Med. 2013;31:1472-8.

9. Dorian P, Cass D, Schwartz B, Cooper R, Gelaznikas R, Barr A. Amiodarone as compared with lidocaine for shock-resistant ventricular fibrillation. N Engl J Med. 2002;346:884-90.

10. Hassan TB, Jagger C, Barnett DB. A randomised trial to investigate the efficacy of magnesium sulphate for refractory ventricular fibrillation. Emerg Med J. 2002;19:57-62.

11. Driver BE, Debaty G, Plummer DW, Smith SW. Use of esmolol after failure of standard cardiopulmonary resuscitation to treat patients with refractory ventricular fibrillation. Resuscitation. 2014;85:1337-41.

12. Lee $\mathrm{YH}$, Lee KJ, Min YH, et al. Refractory ventricular fibrillation treated with esmolol. Resuscitation. 2016;107:150-5.

13. Killingsworth CR, Wei CC, Dell'Italia LJ, et al. Short-acting beta-adrenergic antagonist esmolol given at reperfusion improves survival after prolonged ventricular fibrillation. Circulation. 2004;109:2469-74.

14. Jingjun L, Yan Z, Weijie, Dongdong Z, Guosheng L, Mingwei B. Effect and mechanism of esmolol given during cardiopulmonary resuscitation in a porcine ventricular fibrillation model. Resuscitation. 2009;80:1052-9.

15. Cammarata G, Weil MH, Sun S, Tang W, Wang J, Huang L. Beta1-adrenergic blockade 
during cardiopulmonary resuscitation improves survival. Crit Care Med. 2004;32:

S440-3

16. Spaulding CM, Joly LM, Rosenberg A, et al. Immediate coronary angiography in survivors of out-of-hospital cardiac arrest. N Engl J Med. 1997;336:1629-33.

17. Cheskes $S$, Dorian $P$, Feldman $M$, et al. Double sequential external defibrillation for refractory ventricular fibrillation: The DOSE VF pilot randomized controlled trial. Resuscitation. 2020;150:178-84.

18. Beck LR, Ostermayer DG, Ponce JN, Srinivasan S, Wang HE. Effectiveness of Prehospital Dual Sequential Defibrillation for Refractory Ventricular Fibrillation and Ventricular Tachycardia Cardiac Arrest. Prehosp Emerg Care. 2019;23:597-602.

19. Yannopoulos D, Bartos J, Raveendran G, et al. Advanced reperfusion strategies for patients with outof-hospital cardiac arrest and refractory ventricular fibrillation (ARREST): a phase 2 , single centre, openlabel, randomised controlled trial. Lancet. 2020;396:1807-16.

20. Perkins GD, Ji C, Deakin CD, Quinn T, et al. PARAMEDIC2 Collaborators. A Randomized Trial of Epinephrine in Out-of-Hospital Cardiac Arrest. N Engl J Med. 2018;379:711-21.

21. Mc KW, Gregg DE, Canney PC. Oxygen uptake of the nonworking left ventricle. Circ Res. 1958;6:61223.

22. Berglund E, Monroe RG, Schreiner GL. Myocardial oxygen consumption and coronary blood flow during potassium-induced cardiac arrest and during ventricular fibrillation. Acta Physiol Scand. 1957;41:261-8.

\section{Figures}




\begin{tabular}{|c|c|c|c|}
\hline MEPICINE REFERENCE & Esmolol & $\begin{array}{c}\text { Page 1 of } 1 \\
\text { Last Revision: } \\
02 / 26 / 2020\end{array}$ \\
\hline
\end{tabular}

\section{Pharmacologic Class:}

- Esmolol is a cardioselective beta1 receptor blocker

Indications:

- Refractory VF/VT, defined as VF/VT that persists after epinephrine x 3, defibrillation x 3, and 300mg of Amiodarone

\section{Contraindications:}

- Traumatic Arrest

\section{Dosing:}

\begin{tabular}{c|c}
\hline \multicolumn{1}{c|}{ Adult } & \multicolumn{1}{c}{ Pediatric } \\
\hline - $0.5 \mathrm{mg} / \mathrm{kg}$ Bolus IV $/ \mathrm{IO}$ & \\
\hline Pharmacokinetics: & \\
\hline
\end{tabular}

- Time of Onset: Rapid onset

- Duration: $\sim 9$ minutes

- Half-Life: Rapid distribution half-life of 2 minutes

\section{Side Effects:}

- Hypotension

- Bradycardia

\section{Critical Points:}

- Only indicated for the patient in Cardiac Arrest experiencing Refractory Ventricular Fibrillation and/or Refractory Ventricular Tachycardia.

- Consider limiting Epinephrine administration in the RVF patient

- Use in conjunction with Double Sequential Defibrillation

\section{Figure 1}

Prehospital esmolol for out-of-hospital cardiac arrest with refractory ventricular fibrillation protocol 


\section{Pre-Esmolol}

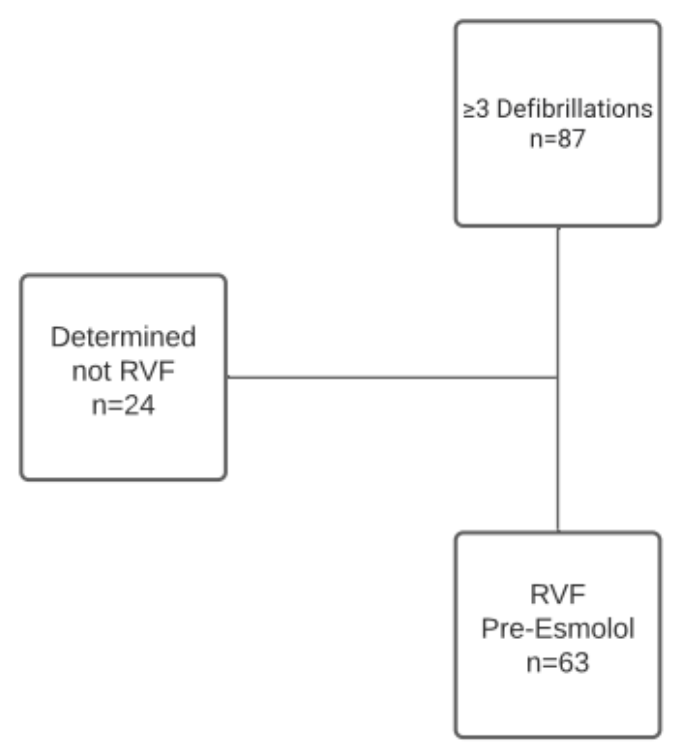

Figure 2

Inclusion of patients in analysis sample

\section{Post-Esmolol}

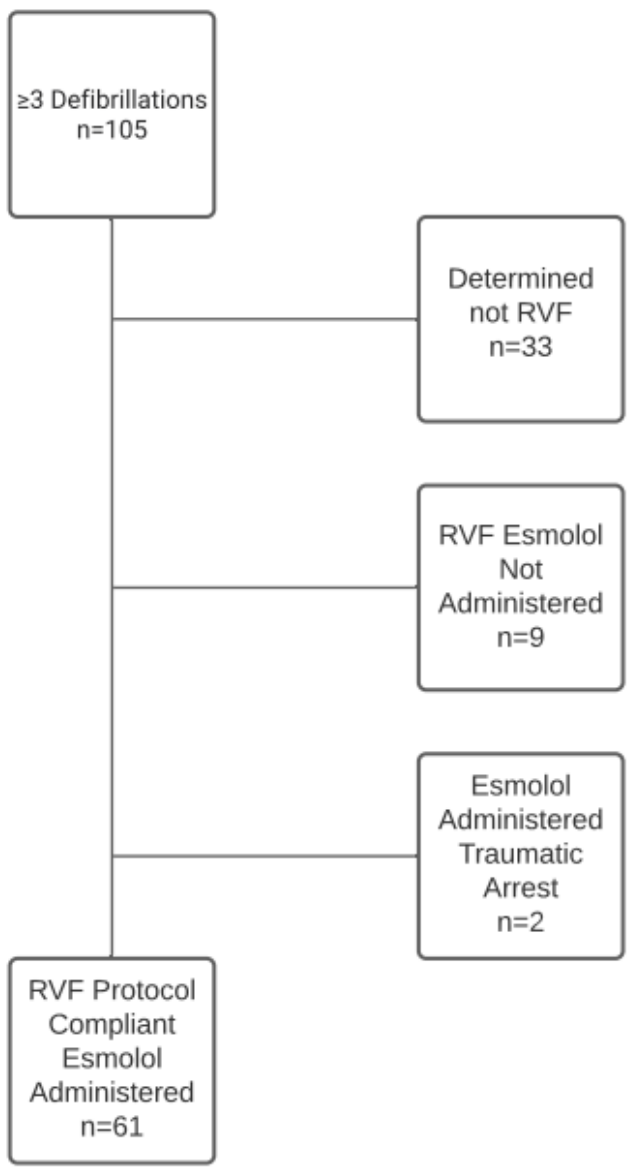




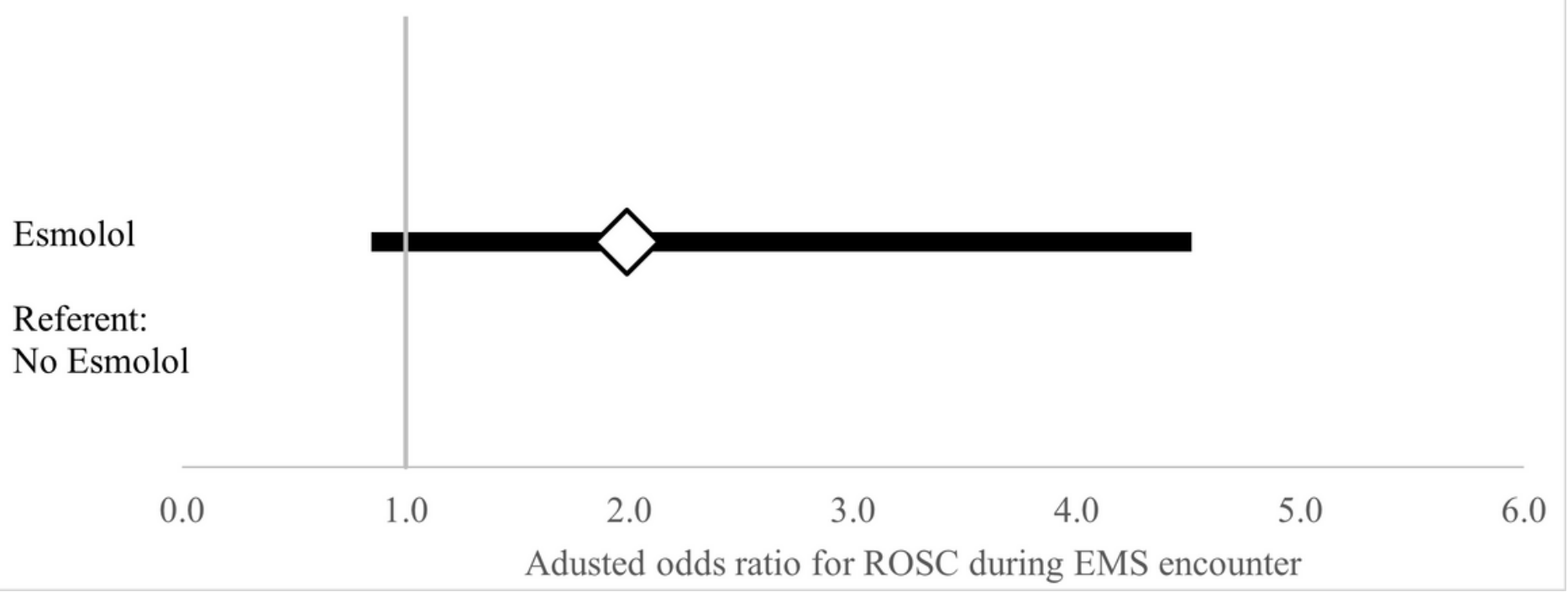

\section{Figure 3}

Odds of ROSC during the EMS encounter for patients administered esmolol versus those who did not receive esmolol, adjusted for age, sex, and initial rhythm. 